\title{
In vivo blockade of OX40 ligand inhibits thymic stromal lymphopoietin driven atopic inflammation
}

\author{
Dhaya Seshasayee, ${ }^{1}$ Wyne P. Lee, ${ }^{1}$ Meijuan Zhou, ${ }^{1}$ Jean Shu, ${ }^{1}$ Eric Suto, ${ }^{1}$ Juan Zhang, ${ }^{1}$ \\ Laurie Diehl, ${ }^{2}$ Cary D. Austin, ${ }^{2}$ Y. Gloria Meng, ${ }^{3}$ Martha Tan, ${ }^{3}$ Sherron L. Bullens, ${ }^{4}$ Stefan Seeber, ${ }^{5}$ \\ Maria E. Fuentes, ${ }^{6}$ Aran F. Labrijn, ${ }^{7}$ Yvo M.F. Graus, ${ }^{7}$ Lisa A. Miller, ${ }^{8}$ Edward S. Schelegle, ${ }^{8}$ \\ Dallas M. Hyde, ${ }^{8}$ Lawren C. Wu, ${ }^{1,9}$ Sarah G. Hymowitz, ${ }^{1,9}$ and Flavius Martin 1
}

\begin{abstract}
${ }^{1}$ Department of Immunology, ${ }^{2}$ Department of Pathology, ${ }^{3}$ Department of Assay and Automation Technology, and ${ }^{4}$ Department of Tumor Biology and

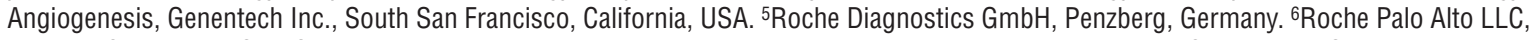
Palo Alto, California, USA. ${ }^{7}$ Genmab B.V., Utrecht, The Netherlands. ${ }^{8}$ Department of Anatomy, Physiology and Cell Biology, School of Medicine, University of California Davis, Davis, California, USA. ${ }^{9}$ Department of Protein Engineering, Genentech Inc., South San Francisco, California, USA.
\end{abstract}

\begin{abstract}
Thymic stromal lymphopoietin (TSLP) potently induces deregulation of Th2 responses, a hallmark feature of allergic inflammatory diseases such as asthma, atopic dermatitis, and allergic rhinitis. However, direct downstream in vivo mediators in the TSLP-induced atopic immune cascade have not been identified. In our current study, we have shown that $\mathrm{OX} 40$ ligand $(\mathrm{OX} 40 \mathrm{~L})$ is a critical in vivo mediator of TSLP-mediated Th2 responses. Treating mice with OX40L-blocking antibodies substantially inhibited immune responses induced by TSLP in the lung and skin, including Th2 inflammatory cell infiltration, cytokine secretion, and IgE production. OX40L-blocking antibodies also inhibited antigen-driven Th2 inflammation in mouse and nonhuman primate models of asthma. This treatment resulted in both blockade of the OX40-OX40L receptor-ligand interaction and depletion of $\mathrm{OX} 40 \mathrm{~L}$-positive cells. The use of a blocking, $\mathrm{OX} 40 \mathrm{~L}$-specific $\mathrm{mAb}$ thus presents a promising strategy for the treatment of allergic diseases associated with pathologic Th2 immune responses.
\end{abstract}

\section{Introduction}

Studies of allergic inflammatory disease pathogenesis such as asthma have shown chronic inflammation resulting from a hyperresponse to innocuous environmental antigens. The pathophysiology of asthma includes mucus hypersecretion, bronchial hyperresponsiveness, smooth muscle hypertrophy, and airway obstruction (1). On a cellular level, the immune response to allergens is mediated by mast cells, $\mathrm{CD} 4^{+}$Th 2 cells, eosinophils, neutrophils, macrophages, and IgE-secreting B cells. Activation and recruitment of $\mathrm{CD}^{+} \mathrm{T}$ cells to sites of Th2 inflammation have been shown to be dependent on cytokines and chemokines produced by antigen-presenting cells as well as costimulation provided by the B7 family and members of the TNF family such as OX40 ligand (OX40L) (2).

Interactions between OX40L and its receptor, OX40, have been shown to be important for regulating effector and memory $\mathrm{CD}^{+}$ $\mathrm{T}$ cell responses $(3,4)$. OX40L is expressed primarily on activated antigen-presenting cells, at low levels on subsets of activated endothelial cells at inflammatory sites and on mast cells involved in chronic GVHD (5). Expression of the receptor, OX40, is observed preferentially on effector and memory $\mathrm{CD}^{+}$and $\mathrm{CD}^{+} \mathrm{T}$ cells. Expression of the ligand and the receptor has also been observed at sites of inflammation in various Th1- and Th2-driven diseases and disease models, including multiple sclerosis, rheumatoid arthri-

Nonstandard abbreviations used: BAL, bronchoalveolar lavage; BALF, BAL fluid; hIgG, human IgG; OX40L, OX40 ligand; TSLP, thymic stromal lymphopoietin. Conflict of interest: D. Seshasayee, W.P. Lee, M. Zhou, J. Shu, E. Suto, J. Zhang, L. Diehl, C.D. Austin, Y.G. Meng, M. Tan, S.L. Bullens, L.C. Wu, S.G. Hymowitz, and F. Martin are employees of Genentech; S. Seeber and M.E. Fuentes are employees of Roche; A.F. Labrijn and Y.M.F. Graus are employees of Genmab; and L.A. Miller, E.S. Schelegle, and D.M. Hyde are employees of UC Davis.

Citation for this article: J. Clin. Invest. 117:3868-3878 (2007). doi:10.1172/JCI33559. tis, inflammatory bowel disease, and asthmatic airways in both human and mouse tissues, indicating a potential role in regulation of autoimmune responses (6). OX40 signaling has been shown to be involved in maintaining primary effector $\mathrm{T}$ cell responses, including clonal expansion, survival, and cytokine secretion (7-9). Memory $\mathrm{T}$ cell responses, and specifically $\mathrm{Th} 2$ responses, have also shown to be regulated by this pathway. Memory cell accumulation at the site of inflammation and reactivation upon antigen exposure were significantly impaired in the absence OX40-derived signaling, indicating that OX40-dependent $\mathrm{T}$ cell costimulation may be critical in driving robust memory $\mathrm{T}$ cell responses (10).

OX40L and receptor knockout mice have defects in antigeninduced $\mathrm{CD}^{+} \mathrm{T}$ cell responses and also have significantly reduced development of Th2 (11) and Th1 diseases $(7,12)$. Conversely, OX40L transgenic mice exhibit elevated numbers of CD4 $4^{+}$effector $\mathrm{T}$ cells and robust increases in disease severity in Th2 models and also in some Th1 pathology $(13,14)$. Neutralization experiments with $\alpha$-OX40L antibodies in various Th1/Th17 (collagen-induced arthritis, experimental autoimmune encephalomyelitis, inflammatory bowel disease) and Th2 (OVA-induced asthma) inflammatory models in vivo have confirmed important roles for this ligandreceptor pair in regulation of disease severity (6). While the above studies suggested that OX40L-OX40 interactions are important for mediating both Th1 and Th2 responses, a recent study by Ito et al. has made a clear distinction in requirements for OX40L between the 2 types of responses (15). They proposed a determinant role for OX40L in promoting Th2 polarization and response of naive $\mathrm{CD}^{+} \mathrm{T}$ cells in the absence of IL-12, while in the presence of IL-12, OX40L served to increase Th1 responses. OX40L-mediated polarization of T cells along the Th2 lineage was initiated by DCs activated with the cytokine thymic stromal lymphopoietin (TSLP). 

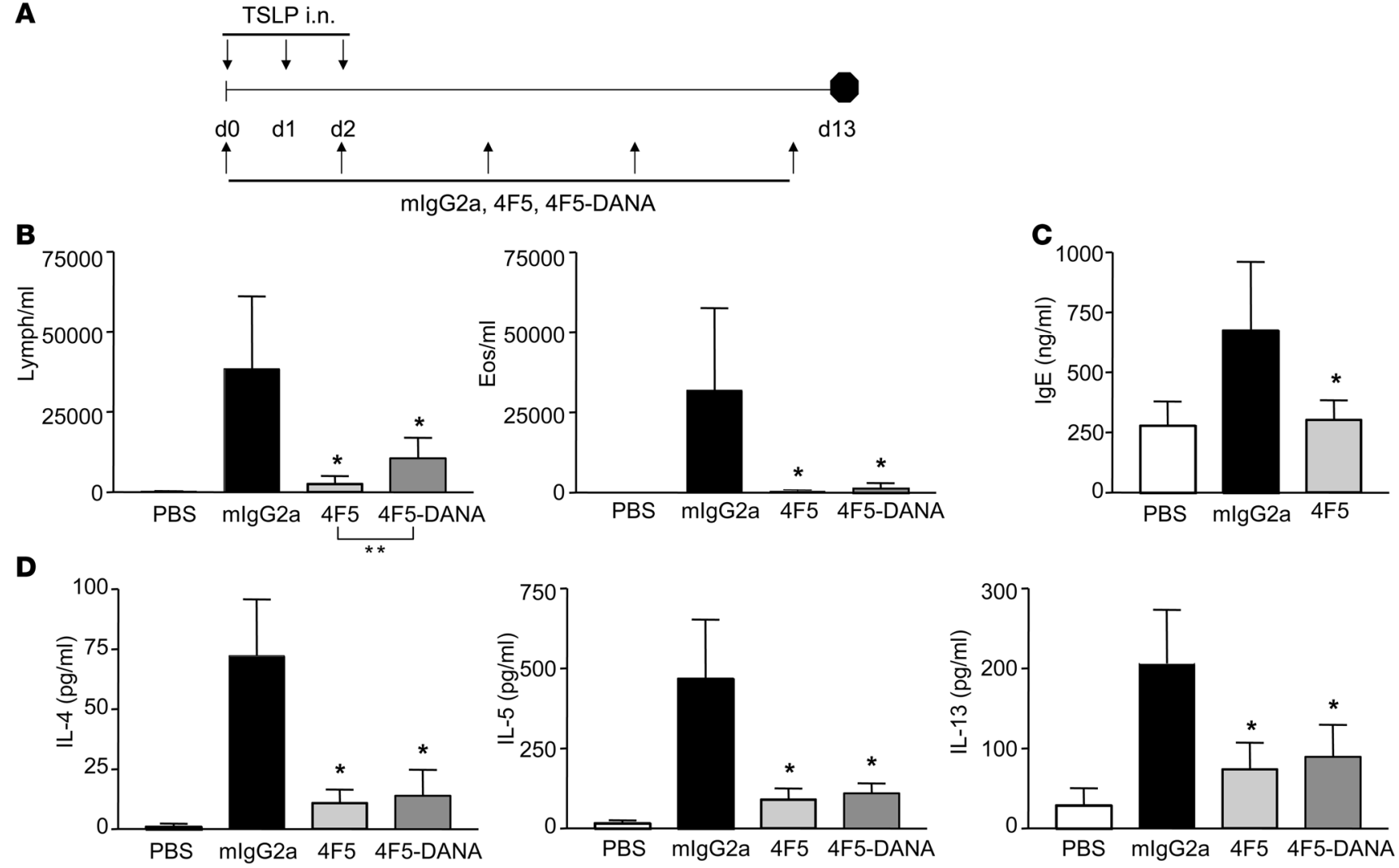

Figure 1

$\alpha$-mOX40L mAb blocks TSLP-induced inflammation in lung. (A) Experimental design for TSLP-induced lung inflammation. BALB/c mice ( $n=8$ /group) were administered $500 \mathrm{ng}$ TSLP (or control PBS) intranasally on days 0,1 , and 2 . Mice were treated with $150 \mu \mathrm{g}$ control mlgG2a, wild-type $\alpha$-OX40L 4F5, or 4F5-DANA mAbs intraperitoneally on days $0,2,5,8$, and 12 and sacrificed on day 13 . Numbers of BALF lymphocytes and eosinophils on day 13 (B) and total IgE levels in serum on day 13 (C) are quantified. (D) Levels of IL-4, IL-5, and IL-13 in the BALF were measured by ELISA. Results are the mean number of cells \pm SD. ${ }^{*} P<0.01$ (Dunnett's test). One representative of 3 experiments is shown.

TSLP is a hematopoietic cytokine whose expression has been detected on crypt epithelial cells in the tonsils, activated pulmonary epithelial cells and fibroblasts, bronchial smooth muscle cells, and IgE-activated mast cells and at high levels at sites of Th2 inflammation, such as epidermal keratinocytes in lesional skin of atopic dermatitis patients and asthmatic bronchial epithelium (16, 17). Recent studies have revealed TSLP to be a potent activator of myeloid DCs, which were shown to secrete Th2-recruiting chemokines TARC and MDC, in addition to IL-8 and eotaxin-2, suggesting that TSLP-activated DCs may represent an initial key step in the development of allergic inflammation $(15,16)$. This concept finds support in studies showing that TSLP-activated DCs can polarize naive $\mathrm{CD}^{+} \mathrm{T}$ cells into Th2 cells secreting IL-4, IL-5, IL-13, and $\mathrm{TNF} \alpha$, and by the reduced disease observed in $\mathrm{TSLPR}^{-/-}$mice in an antigen-induced model of lung inflammation (18). Conversely, transgenic overexpression of TSLP in the lung or skin resulted in spontaneous development of atopic disease in the respective organs. Mice engineered to express TSLP in the lung exhibited a Th2 infiltrate, elevated Th2 responses, airway hyperresponsiveness, and remodeling (18), while inducible TSLP expression in epidermal keratinocytes of the skin mimicked features observed in human atopic dermatitis, including the development of skin lesions with lymphocytic and myeloid infiltrates containing lymphocytes, mast cells, elevated Th2 cytokines, and increased serum IgE (19).
Thus, TSLP appears to be a crucial activator of atopic pathology (20), which mediates its effects through an unknown mechanism distinct from the NF-кB or MyD88 pathways. Recent analyses of microarray experiments on TSLP-stimulated DCs by Ito et al. have shown that OX40L is strongly induced by TSLP (15). Importantly, in vitro neutralization of OX40L activity was able to block TSLP-activated DC polarization of naive $\mathrm{T}$ cells, suggesting that OX40L may be a major mediator of TSLP-induced pathology. Despite this recent body of in vitro data, a dissection of the role of OX40L in TSLP-induced in vivo inflammation has not to our knowledge been performed.

In the present study, we show that an $\alpha$-OX40L-blocking $\mathrm{mAb}$ can significantly inhibit TSLP-induced inflammation in the lung and skin in vivo. Intranasal delivery of TSLP induced a lymphocytic infiltrate accompanied by increases in Th2 cytokines and circulating $\operatorname{IgE}$, all of which were blocked by $\alpha$-OX $40 \mathrm{~L} \mathrm{mAb}$ treatment. Intradermal TSLP delivery into the skin also induced inflammation and Th2 cytokine production, which was effectively blocked by $\alpha-m O X 40 L$ mAb treatment. Efficacy of the mAb was due to a combination of blocking OX40L-OX40 signals and depletion of OX40L-positive cells through Fc receptor-mediated effector function. We have also demonstrated a requirement for OX $40 \mathrm{~L}$ function in a nonhuman primate model of house dust mite (HDM) antigen-induced asthma with a blocking human mAb against 
A
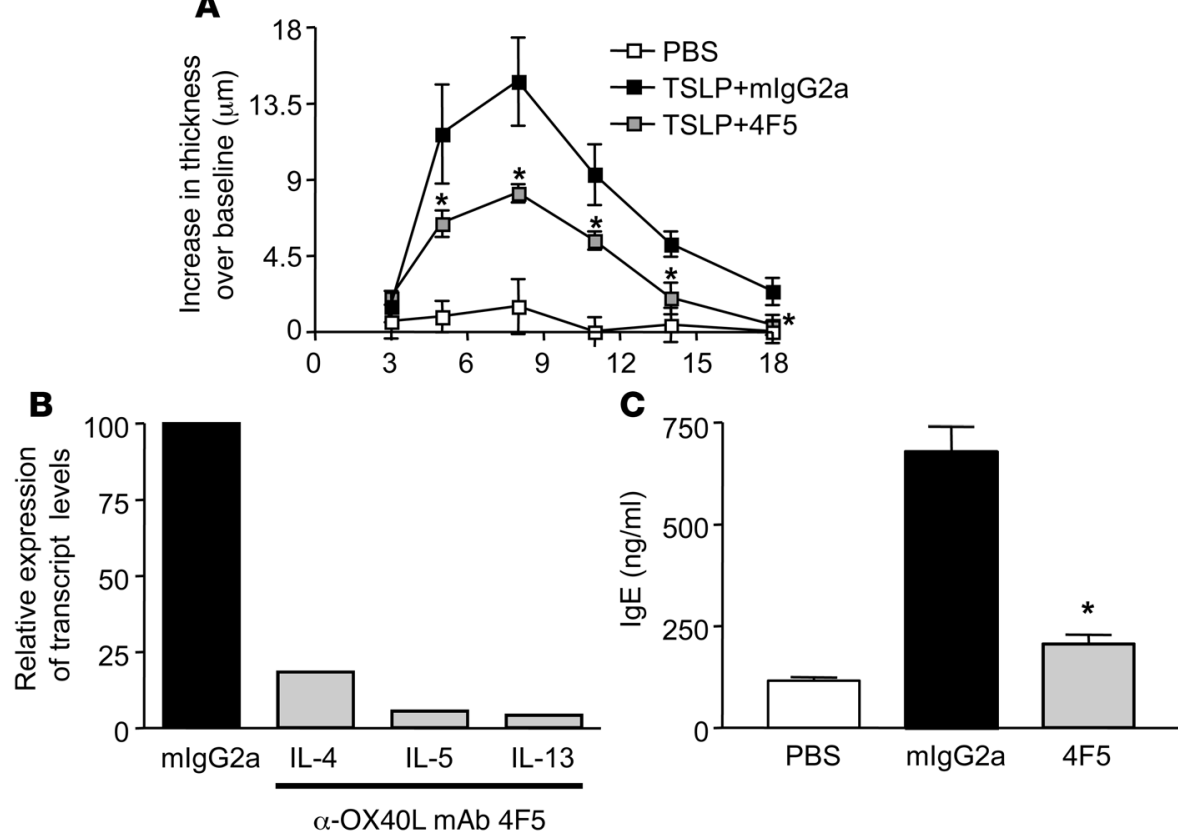

\section{Figure 2}

$\alpha$-mOX40L mAb blocks TSLP-induced inflammation in skin. BALB/c mice $(n=5 /$ group) were administered TSLP or control saline subcutaneously in the ear on days, $0,2,4,7$, and 9 and treated with $150 \mu \mathrm{g}$ mlgG2a or $\alpha$-mOX40L 4F5 mAb twice weekly, starting on day 0. (A) Ear thickness was measured every 3 days, and increase over baseline over the course of the study is shown. (B) Quantitative RT-PCR was performed on RNA isolated from ears of individual mice harvested terminally on day 21 . Effects of $\alpha-O X 40 L$ $\mathrm{mAb}$ treatment on transcript levels of IL-4, IL-5, and IL-13 are shown with control mlgG2a set at a 100. Average Ct values for IL-4, IL-5, and IL-13 for the mlgG2a control group were $26.7,24.2$, and 22.4 respectively, and for the $\alpha$-OX40L mAb treated group, were 29.4, 28.8, and 27.6 respectively. (C) Levels of total serum IgE on day 21 were measured by ELISA and are shown. Results are mean $\pm \mathrm{SD}$. ${ }^{\star} P<0.01$ (Dunnett's test).
hOX40L. Our studies thus demonstrate that OX40L is a critical mediator of TSLP effects in vivo and that inhibition of OX 40L function presents a rational approach for the treatment of allergic and other Th2- linked diseases.

\section{Results}

$\alpha$-OX4OL mAb blocks TSLP-induced lung inflammation. Previous studies have suggested a critical initiating role for TSLP in allergic inflammation (21), and OX40L has recently been identified as a potential downstream mediator of TSLP-induced Th2 responses in vitro (15). To test whether OX40L expression is induced in mouse myeloid DCs upon TSLP stimulation as it is in humans, we activated mouse splenic CD $11 \mathrm{c}^{+}$DCs or human CD $11 \mathrm{c}^{+} \mathrm{DCs}$ (isolated from peripheral blood) with TSLP for 48 hours and examined them for OX40L expression (Supplemental Figure 1, A and C; supplemental material available online with this article; doi:10.1172/JCI33559DS1). In both mouse and human DCs, TSLP was able to potently induce OX40L expression. This induction was the strongest for the myeloid DC subset and was higher than that induced by other stimuli, such as CD40L or TNF- $\alpha$ (data not shown). To determine whether OX40L activity is required for TSLP-mediated effects in vivo, we generated blocking mAbs: a chimeric hamster-mouse mAb, 4F5 (mIgG2a, $\lambda$ ), and a fully human

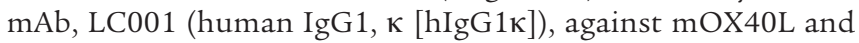
hOX40L, respectively. Blocking abilities of these antibodies were tested in Th2 polarization assays in vitro. As shown in Supplemental Figure 1, B and D, $\alpha$-mOX40L 4F5 mAb and $\alpha$-hOX40L mAb LC001 were able to significantly inhibit Th2 cytokine production compared with the control Ab, confirming that OX40L activity on DCs was important for effects of TSLP on driving Th2 polarization. To test whether OX40L was also a crucial mediator of in vivo TSLP-mediated Th2 inflammation, we measured the ability of blocking $\alpha$-mOX40L 4F5 mAb to inhibit TSLP-induced lung inflammation. BALB/c mice were administered mouse TSLP protein intra-nasally on days 0,1 , and 2 (Figure $1 \mathrm{~A}$ ). Mice were treated with $\alpha$-mOX40L mAbs with (4F5) or without Ab-mediated effector function (4F5-D65A,N297A also called 4F5-DANA) (22) starting on day 0 . Analyses of mouse lungs on day 13 revealed a significant inflammatory infiltrate composed primarily of lymphocytes and eosinophils upon TSLP administration (Figure 1B). Treatment with $\alpha$-mOX40L mAbs (wild type and DANA) significantly inhibited the cellular inflammatory infiltrate. Levels of Th2 cytokines IL-4, IL-5, and IL-13 in the bronchoalveolar lavage (BAL) fluid (BALF), which were induced upon TSLP stimulation, were also significantly inhibited with $\alpha$-mOX40L mAb treatment (Figure 1D), which was accompanied by significant decreases in serum IgE levels (Figure 1C). Inhibition was observed with both wild-type- and effector function-deficient antibodies, suggesting that blocking binding of OX40L to its receptor was sufficient for in vivo efficacy. However, inhibition of lymphocyte number by the wild-type $\alpha$-mOX40L 4F5 mAb was significantly better than the DANA version (Figure 1B), suggesting that effector function may be a component of the increased efficacy of the 4F5 mAb. Importantly, these results clearly indicate a crucial requirement for OX40L function in induction of inflammation by TSLP in the lung.

In order to determine whether delayed blockade of OX40L in this model would still result in significant inhibition of TSLPinduced inflammation, mice were treated with $\alpha-O X 40 \mathrm{~L} \mathrm{mAb}$ beginning on day 0 or day 3 , administered TSLP intranasally on days 0,1 , and 2 , and analyzed for lung inflammation on day 15 (Supplemental Figure 2). As shown, day 3 treatment with $\alpha$-OX $40 \mathrm{~L}$ $\mathrm{mAb}$ had comparable results to that on day 0 , with regard to inhibition of total BALF infiltrate as well as lymphocyte and eosinophil numbers. Levels of IL-4, IL-5, and IL-13 in the BALF were also significantly inhibited. Importantly, total IgE in the serum, which was elevated upon TSLP administration, was also inhibited upon $\alpha$-mOX40L mAb treatment. These observations strongly implicate OX40L as a major downstream mediator of TSLP-induced effects on Th2 responses in vivo.

$\alpha-m O X 40 L m A b$ blocks TSLP-induced inflammation in the skin. To determine whether OX40L function is also important for TSLPmediated effects in the skin, we administered TSLP intradermally 
Unsensitized
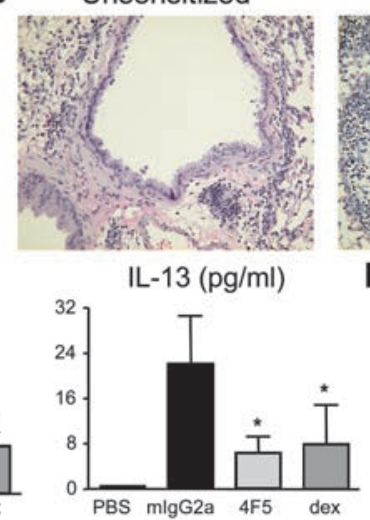

$\mathbf{F}$

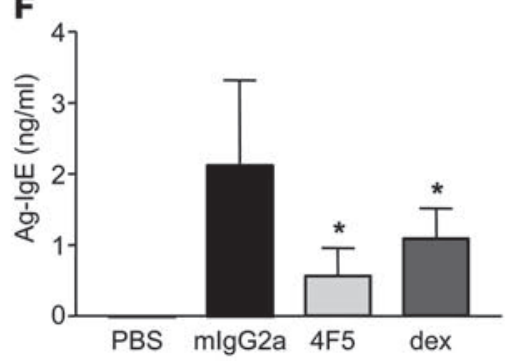

mlgG2a

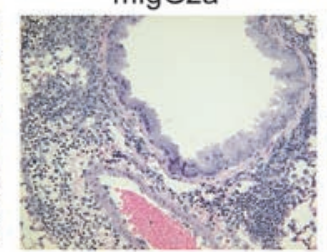

D $\quad \mathrm{Ag}-\mathrm{lgE}(\mathrm{ng} / \mathrm{ml})$
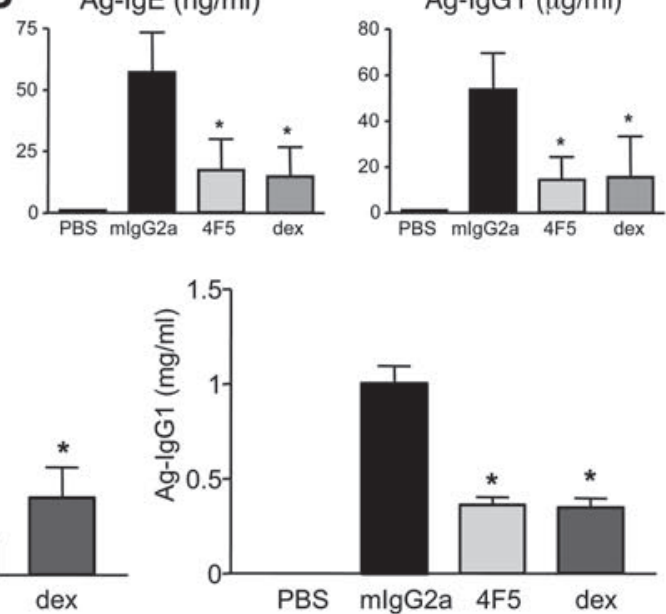

Figure 3

$\alpha$-OX4OL mAb inhibits OVA-induced inflammation in the lung. (A) Experimental design for an acute model of OVA-induced lung inflammation is shown. BALB/c mice (8 per group) were sensitized with OVA/alum or control alum alone on day 0 and challenged with $50 \mu \mathrm{g}$ OVA administered intranasally daily on days $25-28$. Mice were treated with $150 \mu \mathrm{g}$ control mlgG2a, $\alpha-O X 40 \mathrm{~L} 4 \mathrm{~F} 5 \mathrm{Abs}$, or dexamethasone $(2 \mathrm{mg} / \mathrm{kg})$ intraperitoneally on days 26 and 27. Lungs were analyzed for inflammatory infiltrate (B), Th2 cytokines in the BAL (C), and serum antigen-specific IgE and IgG1 levels (D) on day 29. An increased number of OVA challenges model of lung inflammation is shown in E. BALB/c mice were sensitized with OVA/alum (or control alum alone) on day 0, boosted with OVA on day 14, challenged with OVA on days 49-60, and treated with mlgG2a or $\alpha$-OX40L 4F5 antibodies intraperitoneally twice a week or $2 \mathrm{mg} / \mathrm{kg}$ dexamethasone daily, starting on day 53 . Serum antigen-specific $\operatorname{lgE}$ and IgG1 levels were measured on day $74(\mathbf{F})$. Results are mean $\pm \mathrm{SD}$. ${ }^{\star} P<0.01$ (Dunnett's test).

into the ears of BALB/c mice and monitored inflammation with $\alpha-m O X 40 \mathrm{~L} m A b$ treatment. There was a significant increase in ear thickness over baseline in the TSLP-treated group compared with the PBS-treated group, which peaked around day 8 (Figure 2A). Treatment with $\alpha$-mOX40L mAb significantly inhibited the increase in ear thickness. On day 18, RNA was isolated from mouse ears for cytokine analyses. Levels of Th2 cytokines produced in the ear, as assayed by quantitative RT-PCR, were also decreased between 5- and 20- fold after $\alpha$-OX40L mAb treatment (Figure 2B). Additionally, the TSLP-induced increase in total serum IgE assayed at the end of the study was significantly inhibited by $\alpha$-OX $40 \mathrm{~L}$ $\mathrm{mAb}$ treatment (Figure $2 \mathrm{C}$ ). These results indicate that $\mathrm{OX} 40 \mathrm{~L}$ is an important mediator of TSLP-induced pathology in the skin.

$\alpha-m O X 40 L m A b$ inbibits OVA-induced allergic lung inflammation in a chronic mouse model. The above experiments strongly suggest a role for OX40L in TSLP-mediated Th2 pathology in the lung and skin. To determine whether we could use our blocking $\alpha$-OX $40 \mathrm{~L} \mathrm{mAb}$ to inhibit antigen-induced inflammation in vivo, we utilized a mouse model of OVA-induced asthma, which has been shown previously to be dependent on OX40L-OX40 interactions (10). We sensitized mice with OVA/alum, and challenged them with OVA on consecutive days 25-28 (Figure 3A). Control, blocking OX40L antibodies, or dexamethasone were administered after 2 challenges on days 26 and 27. Analyses of inflammatory infiltrate in the lungs by $\mathrm{H} \& \mathrm{E}$ (Figure 3B), total BAL cellularity, and numbers of eosinophils/lym- phocytes (Supplemental Figure 3) indicated that treatment with $\alpha$-OX40L 4F5 mAb was able to inhibit inflammation in the lung compared with the control mIgG2a group. Levels of Th2 cytokines IL-4, IL-5, and IL-13 in the BALF were also decreased significantly (Figure 3C), as were levels of antigen-specific IgE and IgG1 in the serum (Figure 3D). Thus inhibition of OX40L function with blocking of $\alpha$-OX40L 4F5 mAb inhibited OVA-induced disease in an acute model of asthma. However, translation from mouse asthma models to human pathology is limited by lack of chronicity and differences between murine and human lung physiology (23). To start addressing whether $\alpha$-OX $40 \mathrm{~L}$ blocking $\mathrm{mAb}$ would be a therapeutic candidate to inhibit diseases such as asthma, we evaluated a model of OVA-induced inflammation with an increased number of OVA challenges ( 11 versus 4 , as in the acute model discussed above) and also incorporated an OVA boost on day 14 to induce robust memory responses. These changes may allow for a better predictive assessment of the role of OX40L in human asthma. Mice were sensitized with OVA/alum, boosted with OVA on day 14 , and then intranasally challenged with OVA daily for a period of 2 weeks, starting on day 49 (Figure 3E). Treatment with control mIgG2a, $\alpha$-OX40L 4F5 mAb, or dexamethasone was initiated after 5 OVA challenges, when antigen-specific IgE levels peaked, and then continued for a period of 3 weeks. Antigen-specific IgE and IgG1 levels were monitored weekly, and levels on day 74 are shown in Figure 3F. A significant decrease in antigen-specific IgE 

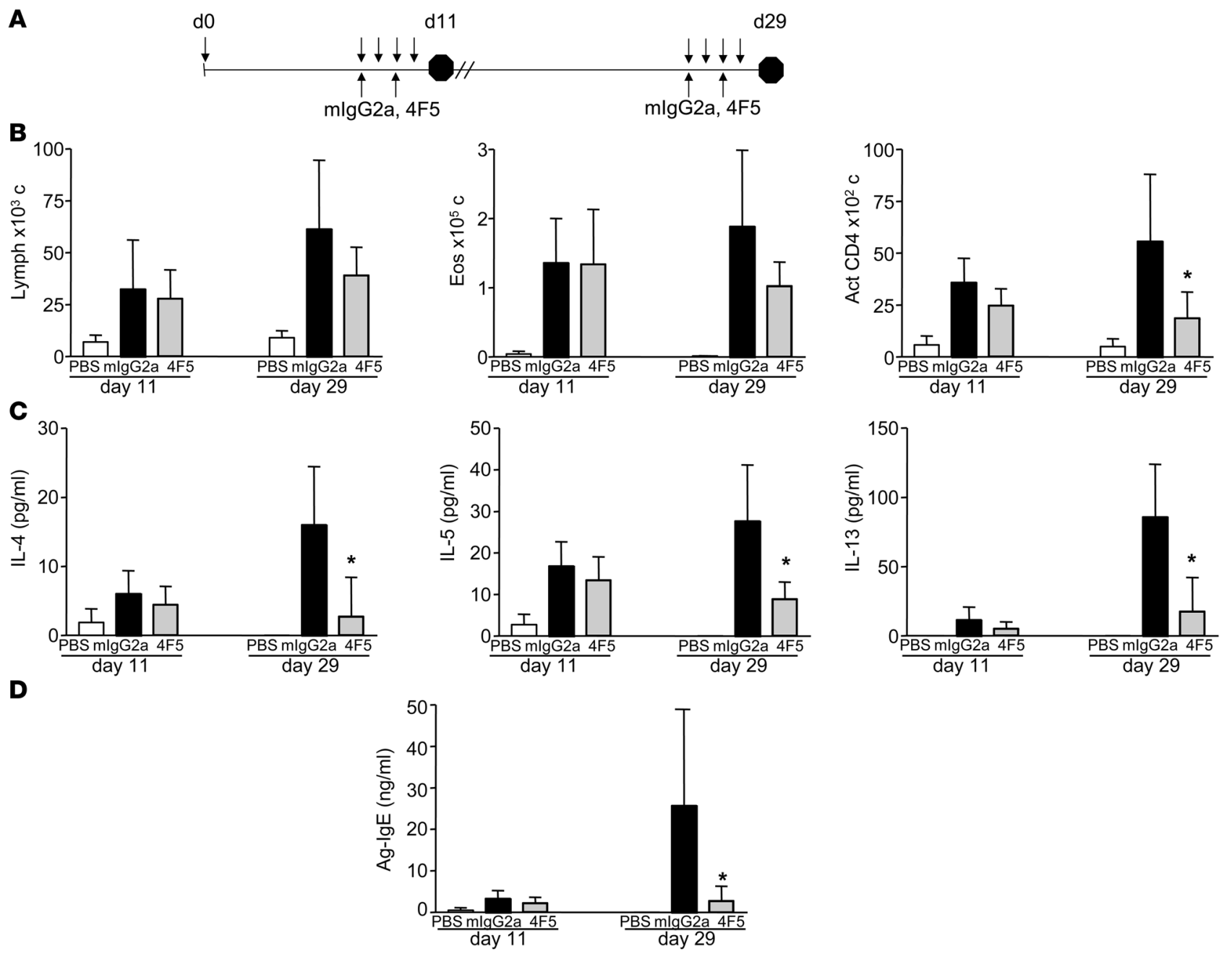

Figure 4

$\alpha$-OX40L mAb inhibits OVA-induced inflammation in the lung mediated by activation of memory T cells. (A) Experimental design for OVA-induced lung inflammation is shown. BALB/c mice ( $n=5$ per group) were sensitized with OVA/alum (or control alum alone) on day 0 . To assay effects of $\alpha$-OX40L mAb treatment on primary effector responses, mice were challenged with OVA intranasally on days 7, 8, 9, and 10 and analyzed for BALF inflammation on day 11. For memory responses, mice were challenged on days 25, 26, 27, and 28 and analyzed on day 29 . Mice were treated with $150 \mu \mathrm{g}$ control mlgG2a and $\alpha$-OX40L 4F5 mAbs intraperitoneally on days 7 and 9 for primary responses, and days 25 and 27 for memory responses. (B) Numbers of infiltrating lymphocytes, eosinophils, and activated CD4+ T cells per ml of BALF on days 11 and 29 are shown. (C) Levels of IL-4, IL-5, and IL-13 in the BALF on days 11 and 29 are shown. (D) Levels of antigen-specific serum IgE are shown. Results are the mean $\pm \mathrm{SD}$. ${ }^{*} P<0.01$ (Dunnett's test).

and IgG1 levels was observed after only 1 week of treatment with $\alpha-O X 40 \mathrm{~L}$ mAb (data not shown), which was only achieved by dexamethasone after 3 weeks of treatment. Similar reductions after $\alpha-\mathrm{OX} 40 \mathrm{~L} \mathrm{mAb}$ treatment were seen when mice were investigated for lung cellular inflammation and BAL Th2 cytokines (data not shown). These results clearly indicate that OX40L interactions are required in vivo for maintenance of Th2 inflammation and that blocking this interaction may inhibit ongoing disease.

Memory T cells producing Th2 cytokines IL-4, IL-5, and IL-13 have been shown to play crucial roles in responding to allergen challenge at sites of allergic inflammation. While the development of these cells during the primary immune response has been well studied, less is known about the regulation and long-term maintenance of these cells. OX40 expression has been detected on both central and effector memory subsets (24), and in vivo studies have revealed the requirement for OX40L signals in the reactivation phase of memory cells upon antigen exposure $(10,11)$. Questions remain, however, regarding the relative importance of roles for OX40 during maintenance of primary effector versus memory responses. To address this issue, we used a model of OVA-induced lung inflammation to test the effect of $\alpha$-mOX $40 \mathrm{~L} \mathrm{mAb}$ on inhibition of primary effector and memory responses to OVA antigen challenge. Mice were sensitized with TNP-OVA/alum on day 0 and challenged with soluble antigen intranasally on days $7-10$ for primary effector responses or days 25-28 for memory responses (Figure 4A). Treatment with $\alpha$-OX40L $4 \mathrm{~F} 5 \mathrm{mAb}$ during the primary effector phase (days 7-9) had little to no effect on maintenance of effector responses as measured by activated $\mathrm{CD} 4^{+} \mathrm{T}$ cell infiltration, Th2 cytokine production, and antigen-specific serum IgE (Figure 4, B-D). In contrast, significant decreases in these param- 


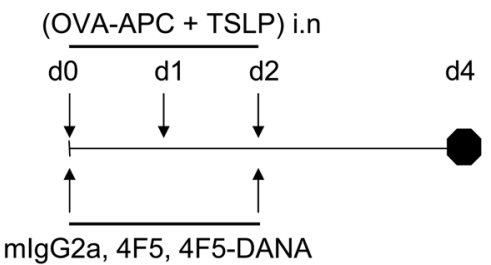

B
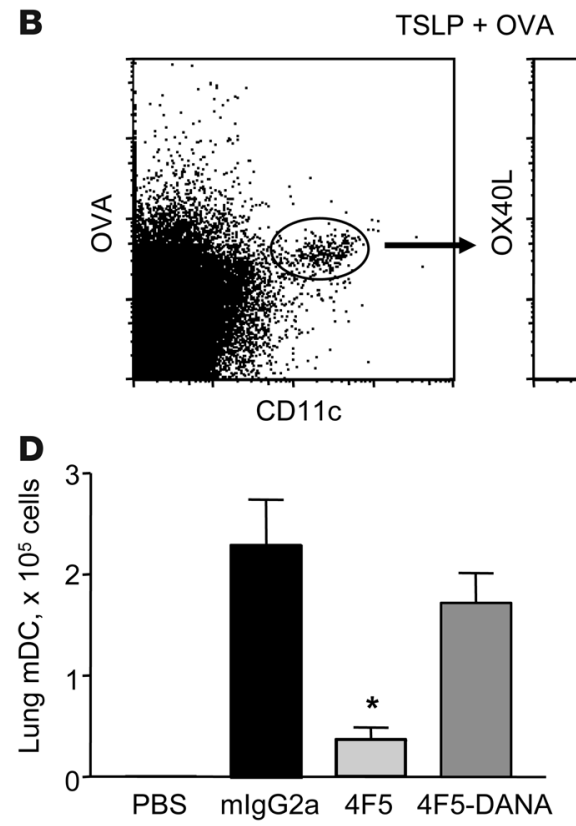

mlgG2a, 4F5, 4F5-DANA

C Saline

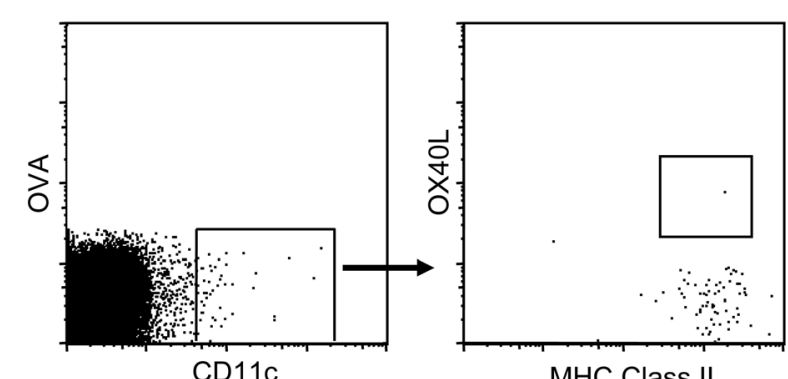

MHC Class II
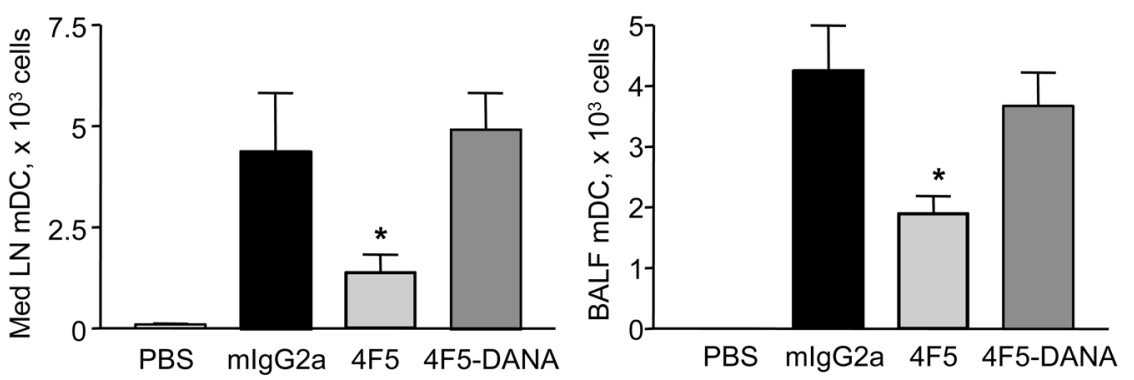

Figure 5

Efficacy of $\alpha-O X 40 \mathrm{~L}$ mAb is mediated in part through depletion of OX40L-expressing DCs. (A) Experimental design to test depletion of DCs by $\alpha-O X 40 \mathrm{~L}$ mAb is shown. BALB/c mice ( $n=6$ per group) were administered TSLP and OVA-APC (or control PBS) intranasally on days 0,1 , and 2 and treated with mlgG2a, 4F5, or 4F5-DANA mAbs on days 0 and 2. On day 4, BALF, mediastinal lymph nodes, and lungs were harvested for analyses of DC numbers. Expression of OX40L on OVA+CD11C+MHC classIl+ DCs in the mediastinal lymph nodes are shown for TSLP and OVA-APC-treated (B) or PBS-treated (C) animals. (D) Total numbers of OVA+CD11C+MHC classll+ DCs in the lung, mediastinal lymph nodes, and BALF are shown. All analyses were performed on live cells (propidium iodide excluded). Results are mean \pm SD and are representative of 2 independent experiments. ${ }^{*} P<0.01$ (Dunnett's test).

eters were observed upon treatment during activation of memory responses (days 25-27). These results suggest that OX40L likely plays a major role in vivo during reactivation of memory responses, as has been observed previously by Salek-Ardakani et al. (10).

$\alpha-m O X 40 L$ mAb blocks OX40L-OX40 interactions and depletes DCs in vivo. In the experiments described above, and in mouse models of OVA-induced asthma, we observed significant in vitro and in vivo efficacy of the $\alpha$-mOX40L 4F5 mAb in inhibiting TSLP-induced inflammation in lung and skin. One question that arises regarding the mechanism of action is the relative contributions of OX40OX40L blockade versus Fc receptor-mediated depletion of OX40Lexpressing DCs. To address this issue, we compared versions of the 4F5 mAb capable of (wild type) or deficient in (DANA) Ab-mediated effector function in multiple models of TSLP-induced lung inflammation (Figure 1B) and OVA-induced allergic inflammation (Supplemental Figure 4). While both versions of the $\alpha$-mOX40L 4F5 mAb were effective in inhibiting inflammation, the wild-type version did exhibit a small but consistent and significant advantage over the effector function mutant in inhibiting several parameters including cellular infiltration and serum antigen-specific IgE and IgG1. This suggested that while the ability to block binding of OX40L to its receptor was a major component of the observed effi- cacy of 4F5, Ab-mediated effector function may have also played a role. In order to test this hypothesis, we compared effects of treatment of 4F5 wild-type and DANA antibodies on numbers of DCs in mice administered a combination of TSLP and APC-labeled OVA (Figure 5A). As shown in Figure 5B, OX40L expression is clearly detected on $\mathrm{OVA}^{+} \mathrm{CD} 11 \mathrm{c}^{+}$Class $\mathrm{II}^{+} \mathrm{DCs}$ in the mediastinal lymph node on day 4 in TSLP-treated mice and is absent in control PBS-treated mice (Figure 5C). The proportion of DCs expressing OX40L ranged from $35 \%-80 \%$ in individual mice. OX40L was also detected on DCs in the lung (data not shown). DC depletion was examined by analyses of total numbers of $\mathrm{OVA}^{+} \mathrm{DCs}$ in control and treated groups in the lung, mediastinal LNs, and BALF (Figure 4C). 4F5 mAb treatment resulted in a significant decrease in the numbers of OVA ${ }^{+}$DCs, which was absent in the 4F5-DANA group, indicating that DC depletion is one of the 4F5 mAb properties and may have contributed to the observed in vivo effect.

To determine whether $\alpha$-mOX40L mAb could also deplete DCs when TSLP was administered through the skin, we treated mice with TSLP in the footpad and analyzed DC numbers in the draining popliteal and inguinal LNs upon 4F5 and 4F5-DANA treatment (Supplemental Figure 5). Similar to the lung, OX40L was detected on CD11 $\mathrm{c}^{+}$DCs in the LNs, and 4F5 treatment 
A Sensitize (wk 0): SQ HDM/alum i.m + i.n.

Boost: SQ HDM/alum + i.n.

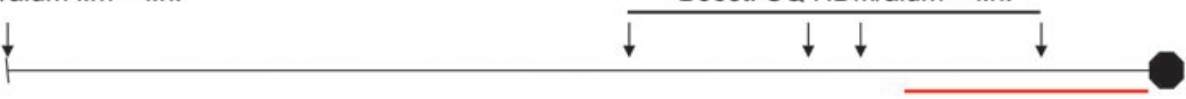

20 wk Rx:

B
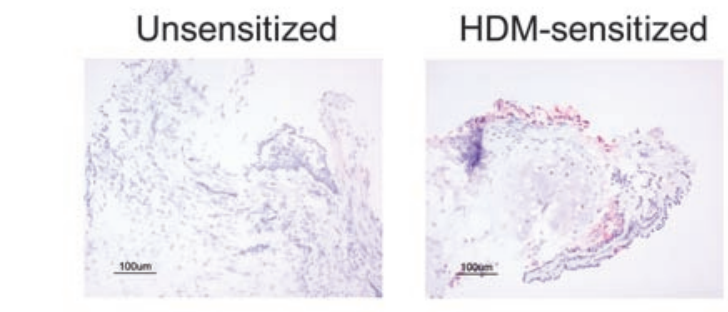

C

$\alpha$-hOX40L mAb, vehicle control
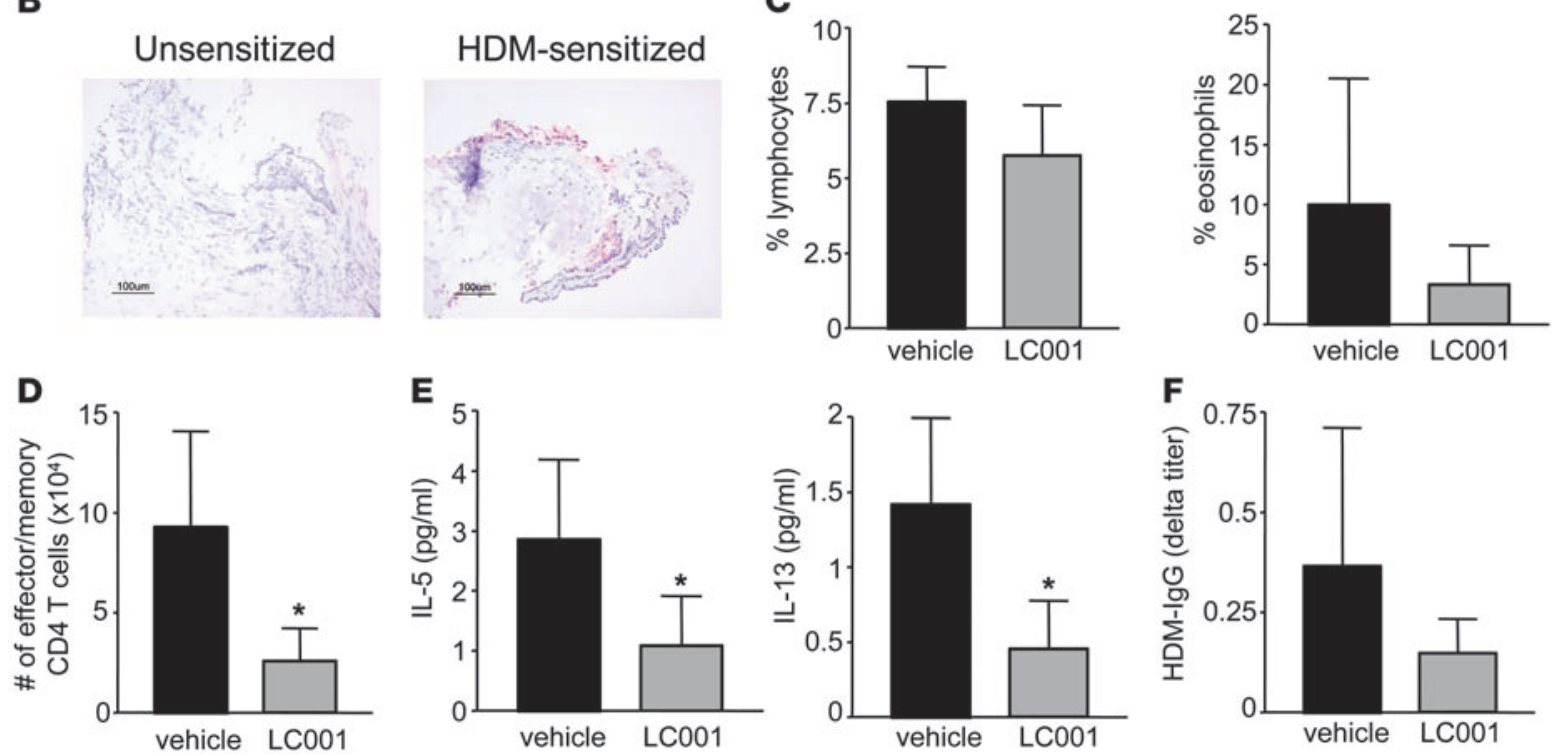

\section{Figure 6}

$\alpha$-hOX40L mAb inhibits HDM-induced asthma in rhesus monkeys. (A) Experimental design for HDM-induced asthma in rhesus monkeys is shown. Young adult monkeys ( $n=6$ per group) were sensitized with HDM/alum and boosted periodically with antigen as shown. Monkeys were treated with $\alpha$-hOX40L mAb LC001 or vehicle control and lung inflammation was analyzed after 22 weeks of treatment. (B) TSLP expression in lung biopsies from unsensitized and HDM-sensitized animals are shown. Percentages of lymphocytes and eosinophils (C), numbers of effector/ memory T cells (D), and levels of IL-5 and IL-13 cytokines (E) in the BALF are shown. Effector memory T cell numbers (CD4 ${ }^{+}$CD45RA-CCR7 ${ }^{-}$) were assayed by FACS with propidium iodide exclusion for live cells. (F) Serum antigen-specific IgG titers ( $\Delta$, week 16-baseline pretreatment) are also shown. Results are mean $\pm \mathrm{SD}$. ${ }^{\star} P<0.01$ (Dunnett's test).

resulted in a significantly lower number of DCs as compared with the mIgG2a and 4F5-DANA groups, indicating in vivo DC depletion by the $4 \mathrm{~F} 5 \mathrm{mAb}$.

$\alpha-b O X 40 L$ mAb inbibits Th2 lung inflammation in nonhuman primates. The above experiments, together with previously published studies $(18,19)$, strongly indicate that TSLP is a major driver of Th2mediated pathology in the lung and skin, and OX40L is a critical downstream mediator in this pathway. Inhibition of OX40L function through the use of a blocking $\mathrm{Ab}$ thus presents an attractive approach for therapy for allergic diseases including asthma and atopic dermatitis. To test this hypothesis in primates, we treated rhesus monkeys enrolled in a model of dust-mite-induced allergic asthma with a blocking human $\mathrm{mAb}$ (LC001) against OX40L (Figure $6 \mathrm{~A})$. To determine whether inflammation in rhesus lungs, similar to humans, is associated with TSLP expression, we stained lung biopsy sections for expression of TSLP protein 9 months after the initial sensitization. TSLP was detected on airway respiratory cells and smooth muscle cells in sensitized monkeys (Figure 6B) but absent in unsensitized monkeys. An increased inflammatory infiltrate was also observed in the respiratory epithelial and lamina propria regions in lung biopsies of HDM-sensitized monkeys (Supplemental Figure 6A) when compared with unsensitized monkeys. OX40L expression was also detected by quantitative RT-PCR in the BAL harvested from monkeys at week 89 (Supplemental Figure 6B). After 18 months of
HDM exposure, monkeys were treated with blocking $\alpha$-hOX 40L mAb LC001 (or vehicle control) for 22 weeks, and their lungs were analyzed for Th2 inflammation. Blocking OX40L function resulted in a slight decrease in total lymphocyte and eosinophil infiltration into the BAL 48 hours after HDM aerosol exposure (Figure 6C). This was accompanied by significant decreases in the numbers of activated effector memory $\mathrm{CD} 4^{+} \mathrm{T}$ cells as well as levels of $\mathrm{Th} 2$ cytokines IL-5 and IL-13 (Figure 6, D and E). IL-4 was not detectable, as has also been observed in human asthmatic lavage specimens (25). Levels of allergen-specific IgG titers (over baseline) were also decreased in LC001-treated monkeys (Figure 6F). These results indicate that treatment with a blocking $\alpha-\mathrm{OX} 40 \mathrm{~L}$ mAb was able to inhibit Th2 inflammation in a nonhuman primate model of asthma.

\section{Discussion}

In the present study, we show that OX40L is a dominant mediator of TSLP-induced allergic inflammation in the lung and skin in mouse models in vivo. We also demonstrate a requirement for OX40L function in antigen-driven models of asthma in mice and nonhuman primate rhesus monkeys. Treatment with blocking $\alpha$-OX40L mAbs led to significant decreases in inflammatory infiltrate comprising eosinophils, lymphocytes, and $\mathrm{CD}_{4}{ }^{+}$effector and memory T cells; Th2 cytokines IL-4, IL-5, and IL-13; and antigenspecific IgE and IgG1. 
Mechanisms of action of the $\alpha$-OX40L mAbs include blocking the binding of OX40L to its receptor OX40 and depletion of OX40Lexpressing cells, including activated DCs. This depletion may be responsible for the increased efficacy of the $\alpha-O X 40 \mathrm{~L}$ mAb with wild-type Ab-mediated effector function, as compared with the deficient DANA mutant. While blocking OX40L function can inhibit Th2 cytokine production and its downstream effects, depletion of the DCs results in direct deprivation of DC-derived activation signals such as cellular costimulation of T cells and additional chemokine production (TARC and MDC) $(15,16)$. In addition to promoting Th2 inflammation, OX40L has also been shown to downregulate IL-10 production by Th2 $\mathrm{T}$ cells and inhibit the generation of IL10-producing $\mathrm{Tr} 1$ regulatory $\mathrm{T}$ cells from naive and memory $\mathrm{CD}^{+} \mathrm{T}$ cells $(26)$. In addition, OX40L was also shown to block the ability of $\operatorname{Tr} 1$ cells to suppress naive $\mathrm{CD}^{+} \mathrm{T}$ cell proliferation. These results indicate an important role for OX40L in breaking tolerance and promoting autoimmune and allergic diseases. Thus our observed efficacy with the $\alpha$-OX40L blocking $\mathrm{mAb}$ in vivo may also involve restoration of peripheral tolerance mechanisms mediated by regulatory $\mathrm{T}$ cells.

Studies with OX40L-blocking reagents $(27,28)$ or in mice lacking OX40L or OX40 receptor expression $(7,9,10,12)$ have provided evidence for an important role for OX40L-OX40 interactions in $\mathrm{CD}^{+}$effector and memory $\mathrm{T}$ cell responses in vivo. Studies by Salek-Ardakani et al. clearly demonstrated a critical role for OX40derived signals in the expansion and maintenance of antigenspecific memory $\mathrm{T}$ cells during recall responses in the lung (10). While these studies have addressed a requirement for OX40 in primary effector and recall responses, questions regarding the relative importance of OX40-derived signals during these 2 phases have not been clearly addressed to date. In our current study, we compare potential inhibitory effects of blocking $\alpha$-OX $40 \mathrm{~L}$ mAbs on these 2 phases in antigen-induced asthma. Administration of the blocking $\mathrm{Ab}$ during a primary effector response had only a moderate effect on Th2 inflammation but during reactivation of memory $\mathrm{CD}^{+}$ responses resulted in a very significant decrease in activated $\mathrm{CD}^{+}$ lung infiltration, Th2 cytokine production, and antigen-specific serum IgE levels, as observed by Salek-Ardakani et al. (10). Thus OX40L may be relatively more important during memory than primary response. These results are inconsistent with a previous study by Hoshino et al. (11), who observed a better suppression of Th2 inflammation upon blocking OX40L function during the sensitization phase, as compared with the challenge phase. The apparent paradox may be due to the differences in study design. In the study by Hoshino et al., mice were immunized on days 0 and 12 and rechallenged on days 22,23 , and 24 , which may have resulted in an elongated effector response from the primary immunization, leading to a decreased $\alpha$-OX40L mAb efficacy. In contrast, in our study, mice were sensitized on day 0 and rested for 24 days before stimulating a memory response. While our results indicate a crucial role for $\mathrm{OX} 40 \mathrm{~L}$ in reactivation of memory responses, additional unpublished results suggest an equally important role in maintenance of memory (D. Seshasayee and F. Martin, unpublished observations). Mice immunized with KLH antigen/alum and treated with blocking OX40L reagents for months 1-4 after challenge had significantly inhibited in vitro $\mathrm{T}$ cell memory reactivation. These data suggest that memory $\mathrm{T}$ cell maintenance is also dependent on OX40L function. These results are in accordance with a recent study by Wang et al. (24), who demonstrated an important role for OX40L on TSLP-stimulated DCs in homeostatic maintenance of human central memory Th2 T cells. Blockade of OX40L function inhib- ited autologous TSLP-DC-induced proliferation of Th2 memory cells by inducing cell cycle arrest at the $\mathrm{G}_{0}$ phase. Results from these human in vitro and mouse in vivo experiments underline the crucial requirement of OX40L-OX40 interactions in the maintenance and reactivation phases of CD4 memory responses.

While our studies have shown a critical role for OX40L in mediating the effects of TSLP on allergic inflammation in vivo, direct effects of TSLP on cells other than DCs have also been shown to be important for the pathology resulting from its overexpression. TSLP receptor expression has been detected on mast cells (29), B cells (30), and activated CD4 ${ }^{+} \mathrm{T}$ cells (31). Activation of human progenitor-derived mast cells with a combination of TLSP and IL-1 $\beta$ or TNF- $\alpha$ resulted in secretion of IL-5 and IL-13 (29). Studies with human $\mathrm{CD}^{+} \mathrm{T}$ cells have demonstrated that TSLP can act in concert with TCR stimulation to drive enhanced proliferation (31). In the murine system, numerous studies have demonstrated direct effects of TSLP on B cell maturation $(30,32)$. In addition, transgenic mice that overexpress TSLP specifically in the skin and develop symptoms resembling atopic dermatitis do not show a significant decrease in disease when crossed with T cell-lacking TCR $\beta^{-/-}$mice (19). These results in combination suggest that, in addition to stimulating Th2 polarization and subsequent activation, TSLP might act directly on myeloid cells to promote allergic responses. Interestingly, mOX 40L expression is upregulated on in vitro IgE-activated, bone marrowderived mast cells (D. Seshasayee and F. Martin, unpublished results) and a blocking $\alpha$-OX40L mAb was able to inhibit mast cell-mediated stimulation of T cells (33). Mast cells have also been shown to produce TSLP upon activation with TNF- $\alpha$. Activated mast cells expressing OX40L and TSLP may thus act to recruit effector and memory $T$ cells to sites of inflammation and subsequently activate these cells to prolong their survival and produce Th2 cytokines. Blockade of OX 40L function may thus inhibit Th2 $\mathrm{T}$ cell activation induced by well-studied mechanisms involving activated DCs as well as by novel mechanisms involving mast cells.

The efficacy of blocking $\alpha$-OX40L mAbs in both murine and nonhuman primate allergen-induced models of asthma indicates a highly conserved role for OX40L in promoting allergic inflammation. Blockade of OX40L function resulted in significant decreases in effector and memory $\mathrm{CD} 4^{+} \mathrm{T}$ cell infiltrate into the lung upon allergen exposure and subsequent Th2 cytokine levels in the BALF in both models. Levels of antigen-specific IgE and IgG1 were also decreased upon treatment with the $\alpha$-OX40L mAb. These observations indicate a crucial role for OX40L-OX40 interactions in Th2 responses, which has been preserved across different species. Identification of TSLP as one of the innate tissue-specific drivers and of OX40-OX40L as a limiting signal in propagating Th2-driven allergic inflammation thus warrants further clinical testing of inhibitors of this pathway in the treatment of the human atopic triad (allergic asthma, atopic dermatitis, and allergic rhinitis).

\section{Methods}

Mice. BALB/c mice (6-8 weeks old) were purchased from The Jackson Laboratory and were housed under specific pathogen-free conditions. All experiments were conducted in accordance with the NIH guidelines for the care and use of mice in research and approved by the Genentech Institutional Animal Care and Use Committee.

Generation of blocking $\alpha-m O X 40 L$ antibodies. Hamsters were immunized with FLAG-tagged recombinant mOX40L (extracellular domain composed of residues 52-198). Antibodies were purified from hybridomas and screened for ability to block binding to OX40 receptor by ELISA using 
plates coated with recombinant $\mathrm{mOX} 40 \mathrm{~L}$ and competition with soluble mOX40 receptor fusion protein. Blocking ability was also confirmed using Chinese hamster ovary $(\mathrm{CHO})$ cells stably expressing full-length mOX40L. Wild-type 4F5 and effector-deficient 4F5-D265A/D297A antibodies were cloned from a hybridoma cell line and a chimera of 4F5 Fv with murine IgG2a heavy chain and $\lambda$-light chain expressed in $\mathrm{CHO}$ cells. To overcome poor solubility issues, a mutant of the 4F5 chimera was constructed, in which an unpaired cysteine in CRD H3 was mutated to serine. The C-to-S 4F5 chimera competed with OX40 receptor and had very similar affinity for $\mathrm{mOX} 40 \mathrm{~L}$ as the parent hamster 4F5. An effector function-deficient version of the $\alpha$-mOX40L mAb, 4F5-DANA, was created by mutating 2 residues (D265A, N297A), which abrogated Fc- $\gamma$ receptor binding (22). All antibodies were purified using protein A chromatography (proSepA) followed by gel filtration chromatography (S200). Protein identities were confirmed by mass spectrometry and $\mathrm{N}$-terminal sequencing. Endotoxin levels in protein used for in vitro and in vivo studies were less than $0.5 \mathrm{EU} / \mathrm{mg}$.

Generation of LC001, a fully human $m A$ b against bOX40L. hIgG transgenic HCo12 mice (Medarex) were immunized with recombinant hOX40L via subcutaneous injections alternating with hOX40L-expressing cells via intraperitoneal injection. (34). Mice were screened for induction of antiOX $40 \mathrm{~L}$ Ab by antigen-specific ELISA and flow cytometric analysis. Hybridomas were generated from spleens and lymph nodes of mice that generated a significant titer against OX40L, and were screened for IgG production and OX40L specificity. Hybridomas were subjected to 2 rounds of limiting dilution cloning, and clones were screened for IgG production, hOX 40L specificity, cross-reactivity with rhesus OX40L, ligand blocking capacity, inhibition of OX40L signal transduction, complement-dependent cytotoxicity, and Fc receptor binding. From this panel, LC001 (IgG1, $\kappa)$ was selected for further development. LC001 heavy and light chains were cloned and expressed in CHO KI1 and purified as described above. The material used in the rhesus study was characterized for percent aggregation $(<0.7 \%)$, endotoxin $(0.26 \mathrm{EU} / \mathrm{ml})$, and protein A levels $(12-14 \mathrm{ng} / \mathrm{ml})$. Final buffer was $10 \mathrm{mM}$ histidine-sulfate, $150 \mathrm{mM} \mathrm{NaCl}$, pH 6.0.

TSLP induction of lung and skin inflammation. Wild-type BALB/c mice were anesthetized and administered $500 \mathrm{ng}$ of recombinant mouse TSLP (R\&D Systems) or control saline intranasally on days 0,1 , and 2 . Mice were treated with $150 \mu \mathrm{g}$ blocking $\alpha$-mOX 40L antibodies in saline intraperitoneal starting on day 0 or 3 . Mice were analyzed for lung inflammation on day 13 or 15 following TSLP administration. For analyses of skin inflammation, BALB/c mice were administered $500 \mathrm{ng}$ of TSLP or control saline subcutaneously in the ear on days, $0,2,4,7$, and 9. Ear thickness was measured every 3 days throughout the study, and ears were harvested for measurement of cytokine transcript levels on day 21. Mice were treated with $150 \mu \mathrm{g}$ blocking $\alpha-\mathrm{mOX} 40 \mathrm{~L} \mathrm{mAb}$ in saline intraperitoneally twice a week starting on day 0 .

OVA-induced lung inflammation. Wild-type BALB/c mice were sensitized on day 0 by intraperitoneal injection of $100 \mu \mathrm{g}$ TNP-OVA (Biosearch Technologies) emulsified in $2 \mathrm{mg}$ aluminium hydroxide in $100 \mu \mathrm{l}$ saline. For nonsensitized control mice, saline plus aluminium hydroxide without OVA was injected. Sensitized mice were boosted with OVA intraperitoneally on day 14. Mice were then challenged intranasally with $50 \mu \mathrm{g}$ OVA daily on days 49-60. Mice were treated with $150 \mu$ g blocking $\alpha$-mOX40L/control mIgG2a antibodies in saline intraperitoneally twice a week, or $2 \mathrm{mg} / \mathrm{kg}$ dexamethasone (Dispensing Solutions Inc.) daily starting on day 53. All mice were euthanized on day 74. For comparison of $\alpha$-OX40L mAb treatment on OVA-induced primary effector and memory responses, mice were sensitized on day 0 by intraperitoneal injection of $100 \mu \mathrm{g}$ TNP-OVA $/ 2 \mathrm{mg}$ alum. For analyses of primary responses, mice were challenged intranasally with $50 \mu \mathrm{g}$ OVA in $50 \mu \mathrm{l}$ saline on days $7,8,9$, and 10 . For memory responses, mice were challenged under with $50 \mu \mathrm{g}$ OVA in $50 \mu \mathrm{l}$ saline on days $25,26,27$, and 28. Mice were treated with $150 \mu \mathrm{g} \alpha$-mOX40L 4F5 or control mIgG2a antibod- ies intraperitoneally in $100 \mu \mathrm{l}$ saline on days 7 and 9 or days 25 and 27, 2 hours prior to OVA challenge. All mice were analyzed 24 hours after the last challenge. For studies of $\alpha$-OX40L mAb-mediated DC depletion, BALB/c mice were administered $1 \mu \mathrm{g}$ TSLP and $800 \mu \mathrm{g}$ OVA-APC (Invitrogen) intranasally on days 0,1 , and 2 and treated with $150 \mu \mathrm{g}$ mIgG2a, $\alpha$-mOX $40 \mathrm{~L}$ 4F5 wild-type, or DANA mAbs intraperitoneally in $100 \mu \mathrm{l}$ saline on days 0 and 2. Mice were sacrificed on day 4, and numbers of DCs in the draining mediastinal lymph nodes, lung, and BALF were assayed.

Characterization of cellular infiltrates. For measurement of infiltrates and cytokines, BAL was performed with $1.5 \mathrm{ml}$ Hank's buffered salt solution (Gibco). Total cell counts were determined using fluorescence beads, and the BALF was centrifuged to remove cells and was used for analyses of IL-4, IL-5, and IL-13 cytokines by ELISA (BD Biosciences - Pharmingen). Cells were used for FACS analyses or cytospin preparations for differential cell counts with a modified Wright-Giemsa stain. After lavage, lungs were perfused with $10 \%$ formalin (Richard Allen Scientific) or OCT (Sakura Fine Technical Company) for pathology analyses.

Antibodies and flow cytometry assays. For analyses of $\mathrm{T}$ cell subsets in BALF, cells were stained with conjugated antibodies to CD4 and CD69 (BD Biosciences - Pharmingen) and CD25 (Miltenyi Biotech) in flow cytometry buffer (PBS with 3\% FBS [Hyclone Laboratories] and $1 \mu \mathrm{g} / \mathrm{ml} \alpha$-CD16/ anti-CD32 [BD Biosciences - Pharmingen]). Expression of OX40L on TSLP-activated mouse DCs was analyzed using a rat $\alpha$-mOX40L mAb (Serotech) conjugated to biotin followed by Streptavidin-PE (BD Biosciences - Pharmingen). Expression of OX40L on human DCs was analyzed using a mouse $\alpha$-hOX 40L mAb 8E12 generated in-house and conjugated to biotin, followed by Streptavidin-PE (BD Biosciences - Pharmingen).

Serum immunoglobulin assays. For measurement of antigen-specific IgE and IgG1 in serum, 96-well Immunosorp plates (Nalgene) were coated with TNPOVA $(5 \mu \mathrm{g} / \mathrm{ml})$ overnight at $4^{\circ} \mathrm{C}$ in a carbonate buffer. Plates were washed, blocked with PBS/10\% FBS (Hyclone Laboratories) for 1 hour at room temperature, washed again, and incubated with serum samples or standards for 2 hours at room temperature. TNP-OVA-specific IgE or IgG1 levels were quantitated using biotinylated rat anti-mouse IgE and IgG1 antibodies (BD Biosciences - Pharmingen). For assays of rhesus serum HDM-IgG, ELISA plates were coated with anti-hIgG Fc (Jackson ImmunoResearch Laboratories). Serum samples were diluted 1:5000 and then further diluted in 3-fold serial dilutions in PBS $\mathrm{pH} 7.4,0.5 \% \mathrm{BSA}, 0.5 \%$ polysorbate 20, 10 ppm proclin. $\mathrm{HDM}-\mathrm{IgG}$ was detected using biotinylated dust mite allergen Der $\mathrm{f} 1$ (Indoor Biotechnologies) followed by HRP-streptavidin (GE Healthcare). Titers were calculated using a cut point, which was set at 6 times the absorbance of the blank rhesus monkey serum (BioReclamation). The dilution at which absorbance value equalled the cut point was calculated from a linear interpolation of absorbance values obtained from serial dilutions of samples. Titer is reported as the $\log _{10}$ of that dilution. Titers for the negative samples were reported as less than 3.7, since a minimum sample dilution factor of 5000 was used.

Quantitative RT-PCR analyses. RNA was isolated from ears with an RNeasy Mini Kit (Qiagen) and was analyzed for IL-4, IL-5, and IL-13 transcript expression using Taqman 1-step RT-PCR Master Mix reagents (Applied Biosystems) with $50 \mathrm{ng}$ of total RNA. Sequences of the primer-probe sets used are as follows: IL-4, forward: GGCATTTTGAACGAGGTCACA, reverse: AGGACGTTTGGCACATCCA, probe: AGAAGGGACGCCATGCACGGAG; IL-5, forward: GGCCACTGCCATGGAGATT, reverse: CTCGGTGAGCGGACAGCT, probe: TGAGCACAGTGGTGAAAGAGACCTTGACA; IL-13, forward: AACGGCAGCATGGTATGGAG, reverse: AGGGAATCCAGGGCTACACA, probe: TGGACCTGGCCGCTGGCG. The probes were conjugated to FAM and TAMRA dyes. Real-time RT-PCR was conducted on an ABI 7500 Real-Time PCR system (Applied Biosystems). Ct values were normalized to RPL-19, a housekeeping gene, and ${ }^{\Delta \Delta} \mathrm{Ct}$ was calculated as $\left({ }^{\Delta} \mathrm{Ct}_{\text {sample }}-{ }^{\Delta} \mathrm{C} \mathrm{t}_{\text {reference }}\right)$. 
Mouse and human DC and T cell assays. For isolation of mouse DCs, BALB/c spleens were digested with $2 \mathrm{mg} / \mathrm{ml}$ collagenase $\mathrm{D}$ (Roche Biochemicals) for 30 minutes at $37^{\circ} \mathrm{C}$. Spleens were then mashed to yield single-cell suspensions, from which CD11 $\mathrm{c}^{+}$DCs were purified using MACS magnetic beads (Miltenyi Biotech). DCs were activated with recombinant mouse $25 \mathrm{ng} / \mathrm{ml}$ TSLP (R\&D Systems) for 48 hours in high-glucose DMEM with 10\% FBS, $2 \mathrm{mM}$ L-glutamine, $100 \mathrm{U} / \mathrm{ml}$ penicillin, and $100 \mathrm{mg} / \mathrm{ml}$ streptomycin and analyzed for OX40L expression by FACS. For mouse Th2 polarization assays, mouse splenic CD $11 c^{+}$DCs were activated with mTSLP for 72 hours, washed, and cultured with OVA-specific DO11.10 CD4 ${ }^{+} \mathrm{T}$ cells (isolated from spleens by negative selection using MACS magnetic beads [Miltenyi Biotech]) in the presence of OVA peptide, IL-2 $(2 \mathrm{ng} / \mathrm{ml}), \mathrm{IL}-4(25 \mathrm{ng} / \mathrm{ml})$, $\alpha$-IL-12 mAb, $\alpha$-IFN- $\gamma$ mAb (R\&D Systems), and $\alpha$-mOX40L 4 F5 or control mIgG2a antibodies for 5 days. Cells were then washed, rested for 3 days with IL-2 $(2 \mathrm{ng} / \mathrm{ml})$, and restimulated with plate-bound $\alpha$-CD3 $(5 \mu \mathrm{g} / \mathrm{ml})$ and soluble $\alpha$-CD28 $(1 \mu \mathrm{g} / \mathrm{ml})$ antibodies for 48 hours. Supernatants were used for IL-4, IL-5, and IL-13 cytokine ELISA analyses (BD Biosciences - Pharmingen). Human myeloid DCs were isolated from peripheral blood mononuclear cells by MACS depletion of CD3-, CD14-, CD16-, CD19-, and CD56-expressing cells, followed by FACS sorting Lin' cells using APClabeled anti-CD11c and a mixture of FITC-labeled mAbs against lineage markers, CD3, CD14, CD16, CD19, and CD56 to reach greater than $99 \%$ purity. Human myeloid DCs were activated with $15 \mathrm{ng} / \mathrm{ml}$ recombinant human TSLP (R\&D Systems) for 48 hours RPMI with 10\% FBS, 2 mM L-glutamine, $100 \mathrm{U} / \mathrm{ml}$ penicillin, and $100 \mathrm{mg} / \mathrm{ml}$ streptomycin and analyzed for OX40L expression by FACS. For human Th2 polarization assays, human TSLP-activated CD $11 c^{+}$DCs were cultured with allogeneic naive $\mathrm{CD}^{+} \mathrm{T}$ cells (isolated from peripheral blood mononuclear cells by MACS negative selection [Miltenyi Biotech]) at a DC/T ratio of 1:4 for 7 days in the presence of IL-2 (5 ng/ml), IL-4 (25 ng/ml), $\alpha$-IL-12 mAb, $\alpha$-IFN- $\gamma$ mAb (R\&D Systems), and $\alpha$-hOX40L LC001 or control hIgG1 antibodies. Cells were then washed, rested for 3 days with IL-2 $(2 \mathrm{ng} / \mathrm{ml})$, and restimulated with plate-bound $\alpha$-CD3 $(5 \mu \mathrm{g} / \mathrm{ml})$ and soluble $\alpha$-CD28 $(1 \mu \mathrm{g} / \mathrm{ml})$ antibodies for 48 hours. Supernatants were used for IL-4, IL-5, and IL-13 cytokine ELISA analyses (BD Biosciences - Pharmingen).

Rhesus model of asthma. A model of allergic asthma in young adult rhesus monkeys (Macaca mulatta) has been previously described (35). Briefly, 12 adult monkeys (housed at the California Regional Primate Research Center, University of California Davis) were sensitized with a subcutaneous injection of $12.5 \mathrm{mg}$ HDM (Dermatophagoides farinae) allergen extract (Greer Labs) in $10 \mathrm{mg}$ alum with $10^{11}$ killed Bordetella pertussis-injected intramuscularly as adjuvant. On day 7 following initial sensitization, monkeys received $94 \mathrm{mg}$ of HDM intranasally. Monkeys were also boosted subcutaneously with HDM/alum and HDM intranasally during weeks 56, 71, 75, and 91. Monkeys were housed in filtered air and exposed to aerosolized HDM (437 $\pm 69 \mu \mathrm{g} / \mathrm{m}^{3}$ protein) for $2-3$ hours twice a week for the length of the study. After 18 months, monkeys (6 per group) were treated with vehicle control or LC001, a fully human mAb against hOX 40L, $7.5 \mathrm{mg} / \mathrm{kg}$ for weeks 1 and 2 and $5 \mathrm{mg} / \mathrm{kg}$ every 2 weeks for the next 20 weeks. Control monkeys were unsensitized rhesus macaques of comparable age that had negative skin tests for HDM allergy. For analyses of BALF, monkeys were lavaged 6 hours (for cytokines and activated T cell readouts) or 48 hours (BALF differential) following aerosol challenge as described previously (35). All aspects of animal work were performed in accordance with institutional guidelines for the California National Primate Research Center and approved by Primate Services Committee at the California Primate Research Center for animal handling, care and coordination, and veterinary care.

Histopathology analyses. Immunohistochemistry and H\&E stains were performed on 5 - $\mu \mathrm{m}$-thick frozen sections of rhesus lung tissue fixed in acetone. The sections were incubated with sheep $\alpha$-TSLP (R\&D Systems), followed by a biotinylated donkey anti-sheep IgG secondary Ab (Jackson ImmunoResearch Laboratories). The $\mathrm{Ab}$ was detected by Vectastain $\mathrm{ABC}$ AP (Vector) and developed with Vector Red (Vector).

Statistics. Statistical tests were performed by Dunnett's test, with single or multiple comparisons with reference to the control group. Significance was assessed using a value of $P<0.01$.

\section{Acknowledgments}

The authors would like to thank Antibody Engineering, Assay and Automation Technology, Protein Purification, and Lab Animal research groups at Genentech, as well as Sarah Davis, Joan Gerriets, and Brian Tarkington at the University of California Davis.

Received for publication August 9, 2007, and accepted in revised form September 26, 2007.

Address correspondence to: Flavius Martin, Department of Immunology, Genentech Inc., 1 DNA Way, MS34, 12-286, South San Francisco, California 94080, USA. Phone: (650) 225-2731; Fax: (650) 225-8221; E-mail: flavius@gene.com.
1. Cohn, L., Elias, J.A., and Chupp, G.L. 2004. Asthma: mechanisms of disease persistence and progression. Annu. Rev. Immunol. 22:789-815.

2. Watts, T.H. 2005. TNF/TNFR family members in costimulation of T cell responses. Annu. Rev. Immunol. 23:23-68.

3. Salek-Ardakani, S., and Croft, M. 2006. Regulation of CD4 T cell memory by OX40 (CD134). Vaccine. 24:872-883.

4. So, T., Lee, S.W., and Croft, M. 2006. Tumor necrosis factor/tumor necrosis factor receptor family members that positively regulate immunity. Int. J. Hematol. 83:1-11.

5. Kotani, A., et al. 2007. Involvement of OX40 ligand+ mast cells in chronic GVHD after allogeneic hematopoietic stem cell transplantation. Bone Marrow Transplant. 39:373-375.

6. Sugamura, K., Ishii, N., and Weinberg, A.D. 2004. Therapeutic targeting of the effector T-cell costimulatory molecule OX40. Nat. Rev. Immunol. 4:420-431.

7. Chen, A.I., et al. 1999. Ox40-ligand has a critical costimulatory role in dendritic cell:T cell interactions. Immunity. 11:689-698.

8. Flynn, S., Toellner, K.M., Raykundalia, C., Goodall,
M., and Lane, P. 1998. CD4 T cell cytokine differentiation: the B cell activation molecule, OX40 ligand, instructs CD4 T cells to express interleukin 4 and upregulates expression of the chemokine receptor, Blr-1. J. Exp. Med. 188:297-304.

9. Gramaglia, I., Weinberg, A.D., Lemon, M., and Croft, M. 1998. Ox-40 ligand: a potent costimulatory molecule for sustaining primary CD4 $\mathrm{T}$ cell responses. J. Immunol. 161:6510-6517.

10. Salek-Ardakani, S., et al. 2003. OX40 (CD134) controls memory $\mathrm{T}$ helper 2 cells that drive lung inflammation. J. Exp. Med. 198:315-324.

11. Hoshino, A., et al. 2003. Critical role for OX40 ligand in the development of pathogenic Th2 cells in a murine model of asthma. Eur. J. Immunol. 33:861-869.

12. Kopf, M., et al. 1999. OX40-deficient mice are defective in Th cell proliferation but are competent in generating B cell and CTL Responses after virus infection. Immunity. 11:699-708.

13. Ishii, N., et al. 2003. OX40 (CD134) and OX40 ligand interaction plays an adjuvant role during in vivo Th2 responses. Eur. J. Immunol. 33:2372-2381.

14. Ndhlovu, L.C., Ishii, N., Murata, K., Sato, T., and Sugamura, K. 2001. Critical involvement of OX40 ligand signals in the $\mathrm{T}$ cell priming events during experimental autoimmune encephalomyelitis. J. Immunol. 167:2991-2999.

15. Ito, T., et al. 2005. TSLP-activated dendritic cells induce an inflammatory $\mathrm{T}$ helper type 2 cell response through OX40 ligand. J. Exp. Med. 202:1213-1223.

16. Soumelis, V., et al. 2002. Human epithelial cells trigger dendritic cell mediated allergic inflammation by producing TSLP. Nat. Immunol. 3:673-680.

17. Ying, S., et al. 2005. Thymic stromal lymphopoietin expression is increased in asthmatic airways and correlates with expression of Th2-attracting chemokines and disease severity. J. Immunol. 174:8183-8190.

18. Zhou, B., et al. 2005. Thymic stromal lymphopoietin as a key initiator of allergic airway inflammation in mice. Nat. Immunol. 6:1047-1053.

19. Yoo, J., et al. 2005. Spontaneous atopic dermatitis in mice expressing an inducible thymic stromal lymphopoietin transgene specifically in the skin. J. Exp. Med. 202:541-549.

20. Liu, Y.J., et al. 2007. TSLP: an epithelial cell cytokine that regulates $\mathrm{T}$ cell differentiation by conditioning dendritic cell maturation. Annu. Rev. Immunol. 
25:193-219.

21. Liu, Y.J. 2006. Thymic stromal lymphopoietin: master switch for allergic inflammation. J. Exp. Med. 203:269-273.

22. Shields, R.L., et al. 2001. High resolution mapping of the binding site on human IgG1 for Fc gamma RI, Fc gamma RII, Fc gamma RIII, and FcRn and design of IgG1 variants with improved binding to the Fc gamma R. J. Biol. Chem. 276:6591-6604.

23. Epstein, M.M. 2004. Do mouse models of allergic asthma mimic clinical disease? Int. Arch. Allergy Immunol. 133:84-100.

24. Wang, Y.H., et al. 2006. Maintenance and polarization of human TH2 central memory T cells by thymic stromal lymphopoietin-activated dendritic cells. Immunity. 24:827-838.

25. Kim, C.K., et al. 2003. Bronchoalveolar lavage cytokine profiles in acute asthma and acute bronchiolitis. J. Allergy Clin. Immunol. 112:64-71.

26. Ito, T., et al. 2006. OX40 ligand shuts down IL-10-pro- ducing regulatory T cells. Proc. Natl. Acad. Sci.U. S. A 103:13138-13143.

27. Higgins, L.M., et al. 1999. Regulation of T cell activation in vitro and in vivo by targeting the OX40OX40 ligand interaction: amelioration of ongoing inflammatory bowel disease with an OX40-IgG fusion protein, but not with an OX40 ligand-IgG fusion protein. J. Immunol. 162:486-493.

28. Yoshioka, T., et al. 2000. Contribution of OX40/ OX40 ligand interaction to the pathogenesis of rheumatoid arthritis. Eur. J. Immunol. 30:2815-2823.

29. Allakhverdi, Z., et al. 2007. Thymic stromal lymphopoietin is released by human epithelial cells in response to microbes, trauma, or inflammation and potently activates mast cells. J. Exp. Med. 204:253-258.

30. Astrakhan, A., et al. 2007. Local increase in thymic stromal lymphopoietin induces systemic alterations in B cell development. Nat. Immunol. 8:522-531.

31. Rochman, I., Watanabe, N., Arima, K., Liu, Y.J., and
Leonard, W.J. 2007. Cutting Edge: Direct action of thymic stromal lymphopoietin on activated human CD4+ T cells. J. Immunol. 178:6720-6724.

32. Vosshenrich, C.A., Cumano, A., Muller, W., Di Santo, J.P., and Vieira, P. 2004. Pre-B cell receptor expression is necessary for thymic stromal lymphopoietin responsiveness in the bone marrow but not in the liver environment. Proc. Natl. Acad. Sci. U. S. A. 101:11070-11075.

33. Nakae, S., et al. 2006. Mast cells enhance T cell activation: importance of mast cell costimulatory molecules and secreted TNF. J. Immunol. 176:2238-2248.

34. Fishwild, D.M., et al. 1996. High-avidity human IgG kappa monoclonal antibodies from a novel strain of minilocus transgenic mice. Nat. Biotechnol. 14:845-851.

35. Schelegle, E.S., et al. 2001. Allergic asthma induced in rhesus monkeys by house dust mite (Dermatophagoides farinae). Am. J. Pathol. 158:333-341. 